\title{
Tacrine Hydrochloride
}

\author{
National Cancer Institute
}

\section{Source}

National Cancer Institute. Tacrine Hydrochloride. NCI Thesaurus. Code C47742.

The hydrochloride salt form of tacrine, an aminoacridine derivative with cog nitive stimulating property. Although the mechanism of action has not been fully elucidated, tacrine hydrochloride may bind reversibly to cholinesterase, acetylcholinesterase as well as butyrylcholinesterase, thereby decreasing the breakdown of acetylcholine, and prolonging synaptic actions as well as increased release of acetylcholine. In addition, this agent inhibits monoamine oxidase (MAO) and may inhibit the reuptake of catecholamines and serotonin. Finally, a novel mechanism of action studied in animal models suggests tacrine to attenuate the production of interleukin-1 beta in the hippocampus and blood, thereby producing central and peripheral anti-inflammatory effects that may play a role in Alzheimer's disease. 